(Aus dem physiologischen Institut der Universität Breslau.)

\title{
Eine Verbesserung der Methode zur optischen Registrierung von Druck und Stromstärke.
}

Von

K. Küurthle.

(Nit 1 Texttigur und Tafel III.)

Bei der früber beschriebenen Methode zur Registrierung von Druck und Stromstärke in den Arterieu ${ }^{1}$ ) war die optische Abbildung dadurch verwickelt, dass Manometer und Stromuhr nicht auf einer, sondern auf zwei parallelen optischen Bänken angebracht waren. Nun kann man ja zwei Instrumente, deren Ansschläge photographisch registriert werlen sollen, auch auf einer optischen Bank hintereinanderschalten, wie dies anerst $\mathrm{B}$ ul1 ${ }^{2}$ ) für die gleichzeitige Registrierung von Elektrokardiogrimm und Herztönen und kürzlich Einthoven ${ }^{3}$ ) mit reinen Sehülern für die gleichzeitige Benützung von zwei Saitengalvanomitern heschieben hat. Allein der Aufstellung von Manometer und Stromulr aluf einer Bank stand die Undurchsichtigkeit der blutgefultten Stromuhr im Wege. Diese lässt sich aber beseitigen, wenu man das Blut am Eindringen in den mit Wasser gefüllten Zylinder verhindert, indem man nach dem Vorgang von Luciani ${ }^{4}$ ) und Trendelenburg ${ }^{5}$ ) Blut und Wasser durch dünne Gummibeutel trennt. Dann bleibt die Stromuhr durchsichtig. Ihr Bild kann nun in der Ehene des Manometerhebels entworfen und diese Ebene wit Hilfe tiner zweiten Linse auf dem Registrier-

1) Pflüger's Arch. Bd. 147 S. 509.1912.

2) Bull, Quart. Journ exper. Phyojol. vol. 4 p. 289. 1911.

3) Pflizger's Arch. Bd. 164 S. 167. 1916.

4) L. Luciani, Physiolngie des Menschen. Deutsch ron Baglioni und Winterstein. Bd. 1 S. 206. 1905.

5) W. Trendelenburg, Eine Stromuhr. Zeitschr. f. Biol. Bd. B5 8. 13. 1015. 
papier abgebildet werden. Mit dieser Anordnung erzielt man ausserdem noch folgenden Vorteil: Während bei der alten Anordnung der Abbildungsraum für beide Apparate getrennt war, indem das untere Drittel des Films von der Druck-, die oberen zwei Drittel von der Stromkurve belegt waren, kann nunmehr die ganze Höhe des Films für beide Kurven ausgenützt, also die optische Vergrösserung der Apparate verstärkt werden, was für die Ausmessung der Kurven von Nutzen ist.

Die Beschreibung der neuen Anordnung zerfällt in die der Stromuhr und der Aufstellung der Apparate auf der optischen Bank.

\section{Die Stromuhr}

unterscheidet sich von dem früher beschriebenen Modell ${ }^{1}$ ) nur hinsichtlich der Zuleitungsröhren, die durch die eingeschalteten Gummibeutel in einen blut- und wasserhaltigen Abschnitt getrennt werden. In Fig. 1 a ist der bluthaltige schraffiert. Die Beutel, die sich abwechselnd aus dem zentralen Ende der Arterie füllen und ins periphere entleeren, liegen in einer Erweiterung der Zuleitungsröhren ( $G l Z$ ) zwischen dem Stromuhrzylinder $(Z)$ und der Drelsscheibe $S c h$, die mit dem Metallgehäuse des Zylinders durch den Stab $S t$ fest verbunden und mit ihm um die Achse $A A$ drehbar ist.

Die Vorrichtung zur Befestigung der Gummibeutel in den $\mathrm{Zn-}$ leitungsröhren ist nicht ganz einfach; zur Klarstellung ist das aus den Teilen $G l Z+T+U+K d$ zusammengesetzte Zwischenstück in Fig. $1 \mathrm{~h}$ in grösserem Maßstab dargestellt: Auf die Drehseheibe Sch sind zu beiden Seiten der Achse $A A$ die kreisförmigen Platten $P l$, aufgelötet, die am Mantel ein Gewinde für die İ̉berwurfmuttern $\ddot{U} M$ tragen. Durch diese werden die Zwischenstücke auf der Drehscheibe festgeschraubt; sie bestehen aus den Glaszylindern $G l Z$ mit ibren gebogenen Fortsätzen $F$ und den Metallmuttern $U$, in welche die Glaszylinder eingekittet sind, und den Metallstücken $T$, welche eine Nute zum Aufbinden der Beutel $G i$ enthalten. Das Aufbinden macht eine Trennung der Metallstücke $T$ und $U$ durch ein Gewinde erforderlich. Um den Gummibeutel (Fingerling für das kleinere Modell, Kondom für das grössere) in der Nute des Stückes $T$ festzubinden, muss das Stück $U$ mit Hilfe eines Schlüssels abgeschraubt werden. In das Stück $T$ ist ferner der haarnadelförmig gebogene Drahtbügel $I B$

1) Pfläger's Arch. Bd. 147 Fìg. 2 S. 514. 
Eino Verbesserung der Methode aur optischen Registrierang usw. 247

eingesetzt, der verhindert, dass der Beutel bei der Tätigkeit der Uhr sich zunächst am festgebundenen Ende entleert und die Öffnung $L$ verstopft. Nach dem Aufbinden der Beutel werden die Stücke $T$ und $U$ wieder verschraubt, so dass die unteren, $P l$ zugekehrten Flächen eine Ebene bilden. Zur Verbindung des Zwischenstückes mit dem Stromuhrdeckel $D$ (Fig. 1a) ist auf den Fortsatz $F$ ein

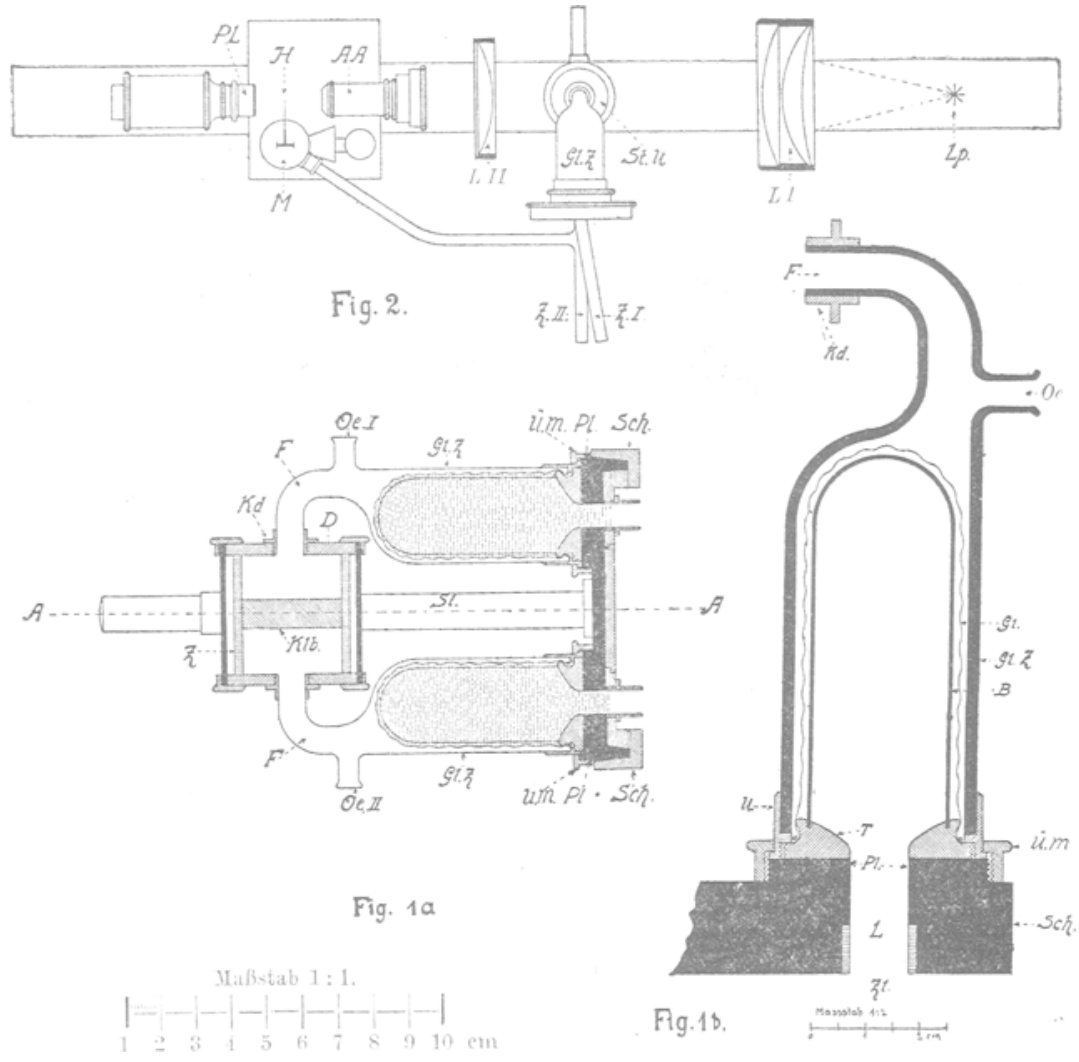

Abschlusskegel ( $K d$ Fir. 1 b) gekittet, der in eine entsprechende Öffnung des Deekels verpanst ist. Ferner ist die der Drehscheibe zugekehrte Fläche von $r+C$ ehen seschliffen und auf die scheibenförmige Erhöhung $P l$ verpasst. Zur Einfügung des zusammengesetzten Zwischenstückes $G l Z+T+U+\mathbb{R} l$ wird dieses derart auf die Drehscheibe geschoben, dass die cenannten ebenen Flächen aufeinandergleiten und der Verschlusskegel $K d$ in die Öffnung des Deckels $D$ tritt. Wird nun die T̈herwurfmutter $\ddot{Z} M$ angezogen, so schliesst die Verbindung dicht, ohne dass der Konus $K d$ eine Ver- 
schraubung erhält. Selbstverständlich müssen die Teile vom Mechaniker sehr genau verpasst und verkittet sein.

Wie beim alten Modell werden die Zuleitungsröhren von der Arterienkanüle bis zum Metallstück $T$ einschliesslich des Bügels $B$ vor dem, Gebrauch mit einer Paraffinschicht überzogen.

Die Füllung der Stromuhr ist etwas umständlicher als beim alten Modell und zerfällt in zwei Abschnitte: Man fülle zunächst den Raum zwischen dem Kolben und den Gummibeuteln, indem man durch die Öffnung OeI Wasser von Körpertemperatur (oder vielleicht besser eine $1 \%$ ige Lösung von oxalsaurem Natron) mit einer Pipette einlaufen lässt, bis der Gummibeutel plattgedrückt ist; dann wird $O e I$ verschlossen, die Stromuhr gewendet und durch Oe II der andere Abschnitt in gleicher Weise gefühlt. Nun werden die Zuleitungsröhren mit den Gummibeuteln gefüllt: Man verbindet die Röhrenmündungen mit grossen, warme Ringer-Lösung enthaltenden Fülllugeln durch weite Gummischläuche und hält die stromuhr so. dass die Füllkugeln den höchsten, die Gummibeutel den tief'sten Funkt einnehmen; zunächst kann keine Lösung eindringen, da die Beutel sich nicht ausdehnen können; man lässt dann durch $O e I$ so viel Wasser abfliessen, bis der Beutel entfaltet, nicht aber elastisch gedehnt ist, und verschliesst $O e I$ wieder. Die Füllung des zweiten Beutels und die vollständige Entfernung der Luft ans den Zuleitungsrönen geschieht durch alwechselndes Heben und Senken der I'üllkugeln bei geeigneter IIaltuns der stromuhr. Dabei zeigt sich dann, dass hei der ersten Füllung des Zylinders noch Luftbläschen zwischen dem Kolben und den Gummibeutelı zurückgeblieben sind, welche nummehr durch die öftnungen Oe $I$ bzw. OeII entfernt werden. Zum Schluss wird der Inhalt beider Gummibentel in die Füllkugeln entleert (indem man durch Oe $I$ oder OeII entsprechend Wasser nachströmen lässt), der Kolben in Endstellung gebracht und nach oben gestcllt, wenn die obere Zuleitungsröhre mit dem zentralen Ende ler Arterie verbunden ist. Lüftet man un nach der Einschaltung der stromuln in die Arterie zugleich mit der Entfernung der zentralen Arterienliemme den Stölsel ${ }^{2}$ ) der Öffnung $O e I I$, so füllt sich der obere Beutel von der Arterie aus, der Kolben wird nach unten getrieben und der Inhalt des Stromuhrzylinder's durch OeII nach aussen entleert. Ist der Kolben am unteren Ende des Zylinders angekommen, so wird OeII geschlossen, und bei der folgenden Wendung der stromuhr ergiesst sich das arterielle Blut in den peripheren Stumpf der Arterie. Man vermeidet so, dass eine erhebliche Menge dor Ringer-Lösung in die Arterie gelangt. Kommt es darauf an, gar keine Ringer-Lösung in die Blutbahn übcrtreten zu lassen, so kann man auch den Inhalt der Zuleitungsröhren durch dic vorgesehenen Öffnungen austreten lassen und durch das einströmende Blut ersetzen.

1) $F_{1}$ und $F_{2}$ in Fig. 2 a S. 514 Pflüger's Arch. Bd. 147.

2) Er lässt sich natürlich auch durch einen Glashahn ersetzen. 


\section{Aufstellung der Apparate auf der optischen Bank.}

Die Anordnung von Stromuhr und Manometer auf der optischen Bank geschieht derart, dass durch eine Linse ( $A A$ Fig. 2) ein verkleinertes Bild der Stromuhr $S t . U$ in der Ebene des Manometerhebels $H$ entworfen und die letztere durch eine zweite Linse $P l$ in die Filmebene projiziert wird. Die Stärke der beiden Linsen ergibt sich aus den folgenden Punkten: 1. Die Höhe der lichtempfindlichen Papierrolle beträgt $12 \mathrm{~cm}$, und diesẻ soll voll ausgenützt werden. Projiziert man den Manometerhebel $H$ auf eine Entfernung von etwa $105 \mathrm{~cm}$, so erhält man durch einen Mikroplanar $F=35 \mathrm{~mm}$ eine annähernd 30 fache Vergrösserung. Da die Ausschläge der abzubildenden Hebelspitze auch bei den grössten Druckschwankungen weniger als $4 \mathrm{~mm}$ betragen, so reicht die Papierhöhe für die Registrierung aller vorkommenden Drucke aus. 2. Die Höhe der benützten Stromuhrzylinder schwankt zwischen 30 und $36 \mathrm{~mm}$; diese braucht aber nicht voll abgebildet zu werden; es genügt eine um die doppelte Kolbendicke geringere Höhe, weil nur die Abbildung der oberen od er der unteren Fläche des Kolbens erforderlich ist. Da dessen Höhe etwa $6 \mathrm{~mm}$ beträgt, kommen von der Gesamthöhe des Zylinders àuch beim grössten Modell nur $24 \mathrm{~mm}$ für die Abbildung in Betracht. Wird nun die Stromuhr durch eine Linse etwa sechsmal verkleinert in die Hebelebene $H$ geworfen. und das verkleinerte Bild durch den Mikroplanar 30 mal vergrössert, so wird die Filmhöhe gerade voll ausgenützt. Der Kolben und seine Bewegung wird dann fünfmal vergrössert auf dem Registrierpapier abgebildet. Zur Verkleinerung: wurde das Z $Z$ is s'sche Objektiv $A A$ (altes Modell) benützt und die Stromuhr in einer Entfernung von $16 \mathrm{~cm}$ aufgestellt, für welche diese Linse korrigiert ist.

Zur Bequemlichkeit der Berechnung der registrierten Kurven wird man Stromuhr und Manometer so vergrössern, dass Druck und Strom dureh gerade Vielfache der. Einheiten dargestellt werden.

Man beginnt nach der Wahl der Linsen mit der Eichung der Stromuhr ${ }^{1}$ ) und reguliert die gewünschte Vergrösserung durch Änderung des Projektionsabstandes. Beim Manometer kann man durch Änderung der Feder- oder Hebellänge ${ }^{2}$ ) leicht den gewünschten Ausschlag erzielen.

1) Pf1üger's Arch. Bd. 147 S. 516.

2) Pflüger's Arch. Bd. 147 S. 512 (3). 
Bildet man nun auf diese Weise den Kolben $a b$, während er sich in verschiedenen Höhen der Stromuhr befindet, und das Objektiv $A A$ auf den ihm zugekehrten Rand des Kolbens eingestelit ist, so erhält man Bilder von verschiedener Höhe und von wechselnder Schärfe. Benützt man einen Hartgummikolben, so sind seine Ränder nur in der Mittellage (optischen Achse) scharf, und seine Höhe scheint von hier aus nach beiden Richtungen zu wachsen. Benützt man einen Glaskolben mit eingeritzter Mantellinie, so erscheint die Linie nur in der optischen Achse einfach, bei der Entfernung aus dieser teilt sie sich in zwei, eine scharf abgebildete und eine unscharfe. Die Kanten des Kolbens erseheinen nur in der optischen Achse scharf. Diese die Ausmessung der Kurven störende Erscheinung hat ihren Grund darin, dass durch das Objektiv $A A$ nicht ausschliesslich der vordere Rand des Kolbens abgebildet wird, sondern der ganze Kolben in perspektivischer Verkürzung, und zwar so, wie er einem in der Eintrittspupille des Objektivs liegenden Auge erscheinen würde. Um diesen Mißstand zu beseitigen, muss man das Zentrum der Perspektive ins Unendliche verlegen. Eine solche Änderung lässt sich dadurch erzielen, dass man zwischen Stromuhr und Objektiv eine Kollektivlinse $L_{I I}$ (Fig. 2) bringt, deren Brennpunkt mit der Eintrittspupille das Objektivs zusammenfällt. Eine diesem Zweck entsprechende Linse ist $\%$. B. die Linse $I$ des lichtstarken Sammellinsensystems für Mikroprojektion von Zeiss ${ }^{1}$ ) mit etwa $15 \mathrm{~cm}$ Brennweite. Die Aufstellung der Apparate gestaltet sich dann so, wie sie in Fig. 2 S. 247 skizziert ist: $L p$ ist eine Bogenlampe von 5 Amp. Stromstärke, deren Strablen durch die Sammellinse $L_{I}$ parallel gemacht werden und die Stromuhr StU treffen; diese ist der linse $L_{I I}$ so weit genähert, dass sie bei der Wendung die Linse nicht berührt. Die Verbindung des Manometers $(\boldsymbol{M})$ mit der Stromuhr zum Zweck der gleichzeitigen Registrierung von Druck und Stromstärke geschieht in der früher ${ }^{2}$ ) geschilderten Weise und ist aus Fig. 2 ersichtlich. Es wird der Seitendruck der peripheren Zuleitungsröhre $Z_{I I}$ registriert, so dass der in der Stromuhr auftretende Druckverlust bei der Bemessung der treibenden Kraft des Stromes nicht berücksichtigt werden muss. Zur Bestimmung der angewandten Vergrösserungen bringt man an Stelle der Vorderfläche des Stromuhrkolbens einen auf Glas ge-

1) Siehe Köhler, Zeitschr. f. Mikrosk. Bu. 19 S. 417. 1902.

2) Pfilüger's Arch. Bd. 147 S. 508. 
ritzten Maßstab, einen zweiten in die Ebene des Manometerhebels $H$ und bildet beide nacheinander oder gleichzeitig ab. Bei einem Versuch, bei welchem die Aufstellung derart erfolgte (Projektionsdistanz $105 \mathrm{~cm})$, dass an der Stromuhr bei einem Durcbfluss von $10 \mathrm{ccm}$ die registrierte Kolbenbewegung $100 \mathrm{~mm}$ und am Manometer für einen Druckzuwachs von $10 \mathrm{~cm}$ Wasser die Ordinate $4 \mathrm{~mm}$ betrug, ergab sich, dass die Stromuhr 4,87 fach, der Manometerhebel 28,5 fach vergrössert wurde.

Dass die registrierten Kurven an Deutlichkeit den nach der alten Anordnung gewonnenen ${ }^{1}$ ) nicht nachstehen, zeigen die Kurven der Taf. III; sie stellen Druck und Stromstärke in der rechten Cruralis eines Hundes von $15 \mathrm{~kg}$ dar, und zwar zu Beginn der Adrenalinwirkung (3 Tropfen i:1000). Man sieht, dass Strom- und Druckkurven sich teilweise überlagern; doch wird die Deutlichkeit des Verlaufs der einzelnen dadurch sehr wenig beeintrïchtigt. Dagegen wird die quantitative Verwertung der Kurren durch die Grösse der Ausschläge nicht unwesentlich verbessert. Auch sieht man an den Druckkurven deutlich die Nebenwellen der Aortenkurve (die Vorschwingungen, den Knick im aufsteigenden Schenkel und die Inzisur), die nach $\mathrm{Frank}$ in der Cruralis nicht vorkommen sollen ${ }^{2}$ ).

Die dritte Kurve $\left(I^{\prime} 7\right)$ stellt das Plethysmogramm der blossgelegten linken Cruralarterie dar, das für einen bei anderer Gelegenleit zu besprechenden Zweck aufgenommen wurde. Iie Bestbreibung des Plethysmographen wird gleichfalls später erfolgen.

Beim Vergleich der Strom- und Druckkurven fällt auf, dass der Anstieg der Strom- wegen die Druckkurve merklich verspätet ist, eine Erscheinung, welche auch bei der alten Anordnung der Apparate auf doppelter Bank schon bemerkt und durch den Unterschied der Eimpfindlichkeit von Manometer und Stromuhr erklärt wurde ${ }^{3}$ ). I)amals musste nachgewiesen werden, dass die Verspätung nicht durch eine parallaktische Verschiebung der Kurven bedingt ist.

Bei diesem Versuch entspricht einem Durchfluss von $10 \mathrm{cem}$ durch die Stromubr eine Ordinate von $70 \mathrm{~mm}$ und einem Druckzuwachs von $10 \mathrm{~cm}$ Wasser eine Ordinate von $3 \mathrm{~mm}$. Die Abszisse chtspricht einem Druck von $20 \mathrm{~cm}$ Wasser.

1) Pflüger's Arch. Bd. 147 Taf. $1 X$ und Bd. 162 Taf. II -V.

2) bie sind nicht eine Folge der Adrenalinwirkung, sondern schon in der nor'malen Kurve ansgebildet.

3) Pflügcr's Arch. Bd. 162 S. 321. 


\section{Feststellung des Stromuhrwiderstandes.}

Obwohl von vornherein anzumehmen ist, dass durch die dünnen Gummibeutel keine erhebliche Vermehrung des Stromuhrwiderstandes geschaffen wird, habe ich doch eine Prüfung ausgeführt und ihr Ergebnis mit dem eines ähnlichen Versuches verglichen, der früher an einer Stromuhr ohne Trennung des Inhaltes der Zuleitungsröhren durch Gummibeutel erhalten worken war.

Zur Bestimmung des Widerstandes wurde destilliertes Wasser von Zimmertemperatur unter bekanntem, konstantem Druck durch geeichte Widerstände ${ }^{1}$ ) teils ohne, teils mit Einschaltung der Stromuhr geleitet und der Stromverlust festgestellt, der im letzteren Falle eintrat. Das Ergebnis dieser Versuche ist in Tabelle I zusammengestellt.

Tabelle I.

\begin{tabular}{|c|c|c|c|c|c|}
\hline \multirow{2}{*}{$\begin{array}{c}\text { Iruck } \\
\text { (cm } \\
\text { Wasser) }\end{array}$} & \multirow{2}{*}{$\begin{array}{l}\text { Sek.- } \\
\text { Volum } \\
\mathrm{ccm}\end{array}$} & \multicolumn{2}{|c|}{$\begin{array}{l}\text { Sek.-Volum } \\
\text { pro Druckeiuheit }\end{array}$} & \multirow{2}{*}{$\begin{array}{c}\text { Strom- } \\
\text { verlust } \\
\%\end{array}$} & \multirow{2}{*}{ Bemerkungen } \\
\hline & & $\begin{array}{c}\text { ohne } \\
\text { stromuhr }\end{array}$ & $\begin{array}{l}\text { mit } \\
\text { Stromıhr }\end{array}$ & & \\
\hline $\begin{array}{l}82 \\
78 \\
92\end{array}$ & $\begin{array}{l}1,3 \\
2,8 \\
3,8\end{array}$ & $\begin{array}{l}0,0166) \\
0,0 ; 16 i \\
0,0445\end{array}$ & $\begin{array}{l}0,0157 \\
0,0295 \\
0,0410\end{array}$ & $\begin{array}{l}5,4 \\
6,7 \\
7,9\end{array}$ & 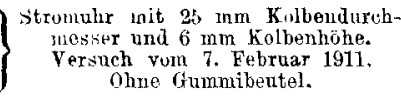 \\
\hline $\begin{array}{l}80 \\
80 \\
80\end{array}$ & $\begin{array}{l}1,3 \\
1,7 \\
2,1\end{array}$ & $\begin{array}{l}0,0165 \\
0,0222 \\
0,0280\end{array}$ & $\begin{array}{l}0,0157 \\
0,0212 \\
0,0265\end{array}$ & $\begin{array}{l}4,9 \\
5,0 \\
5,4\end{array}$ & $\begin{array}{c}\text { Stromuhr nit } 35,7 \mathrm{~mm} \text { Kolbendureh- } \\
\text { messer und } 8,5 \text { mm Kolbenhöhe. } \\
\text { Versuch yom 2. September } 1916 \text {. } \\
\text { Mit Gummibeutel. }\end{array}$ \\
\hline
\end{tabular}

Die Tabelle lehrt, dass der Stromverlust etwa $5 \%$ beträgt, und dass er bei dem Modell ohne Gummibeutel sogar etwas grösser ist. Das ist zum Teil auf die etwas größseren Stromstärken, zum Teil aber vermutlich auf den geringeren Durchmesser des Kolbens zurückzuführen, dcr einen entsprechend grösseren Weg zurückzulegen hat und dadurch etwas grössere Reibung veranlasst.

In einer zweiten Versuchsreihe wurden die durch die Stromuhr veranlassten Druckverluste in folgender Weise hestimmt: Durch die Stromulır und die angeschlossenen Widerstände wurde wiederum destilliertes Wasser geleitet. Vor der zentralen und hinter der peri-

1) Ptlüger's Arch. Bd. 147 Fig. 3 S. 541. In den Versuchen der Tabelle I rom 7. Februar 1911 und rom 2. September 1916 wurden nicht die gleichen Widerstände verwendet. 
pheren Stromuhrkanüle (von $4 \mathrm{~mm}$ Durclimesser i. L.) waren T'-Röhren eingeschaltet, deren freie Schenkel mit den Schenkeln eines Differentialwassermanometers verbunden waren. Dieses bestand aus einer haarnadelförmig gebogenen, senkrecht aufgestellten Röhre von $30 \mathrm{~cm}$ Länge, an deren Biegungsstelle ein Glashahn eingeschmolzen ist. Lässt man bei geöffnetem Hahn in das untere Drittel der Röhren Wasser eindringen und schliesst danu den Hahn, so ist der Druckverlust am Höhenunterschied der in beiden Schenkeln stehenden Flüssigkeitssäulen ohne weiteres abzulesen. Das Ergebnis dieses Versuches ist aus Tabelle II zu erkennen.

$$
\text { Tabelle II. }
$$

Druckverluste in der Stromuhr mit $35,7 \mathrm{~mm}$ Kolbendurchmesser.

\begin{tabular}{c|c|c}
$\begin{array}{c}\text { Druck } \\
\text { (cm Wasser) }\end{array}$ & $\begin{array}{c}\text { Stromstärke } \\
\text { ccm/sec }\end{array}$ & $\begin{array}{c}\text { Druckveriust } \\
\text { (cm Wasser) }\end{array}$ \\
\hline & & 0,9 \\
80 & 1,3 & 1,0 \\
80 & 1,7 & $\mathbf{1}, 5$ \\
80 & 2,1 & 1,6 \\
150 & 2,3 & 2,5 \\
150 & 3,2 & 3,5 \\
150 & $\mathbf{4 , 0}$ &
\end{tabular}

Die Tabelle zeigt, dass die Druckverluste auch bei Stromstärken, wie sie in der Karotis sehr grosser Hunde nur ausnahmsweise vorkommen, recht unerheblich sind. Die durch den Widerstand der Stromuhr veranlassten Fehler können also als sehr geringfügig bezeichnet werden.

Dass die Stromuhr auch den pulsatorischen Stromverlauf richtig darstellt, ist schon früher ${ }^{1}$ ) durch besondere Versuche nachgewiesen worden.

1) Pflüger's Arch. Bd. 147 S. 553. 


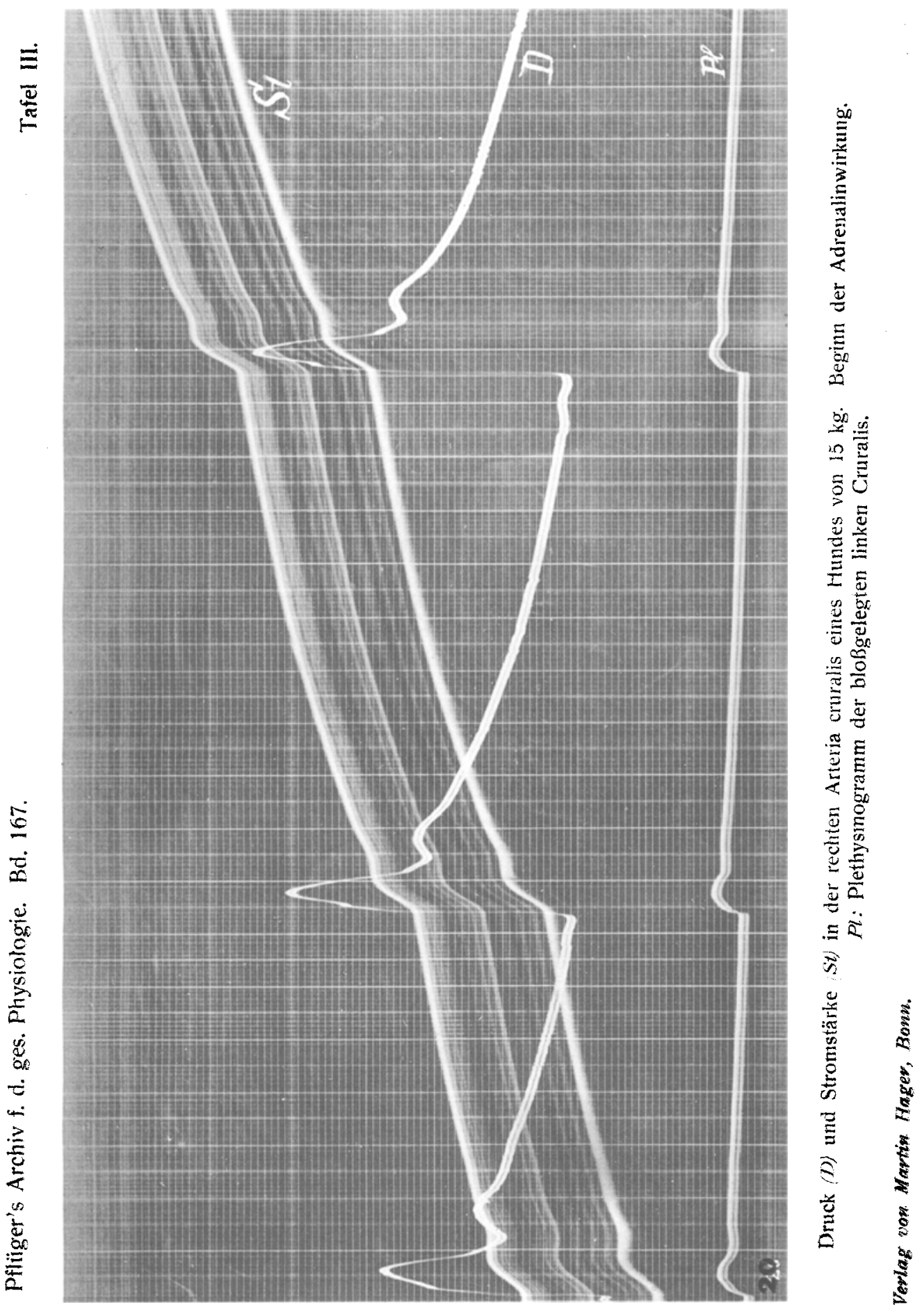

